\title{
LA RELACIÓN DE RESPONSABILIDAD ENTRE EL ESTADO Y LOS PROVEEDORES DE SERVICIOS DE TELECOMUNICACIONES*
}

\author{
Jairo Andrés Becerra Ortiz**
}

Fecha de recepción: 5 de agosto de 2014

Fecha de evaluación: 4 de noviembre de 2014

Fecha de aprobación: 18 de agosto de 2015

Artículo de reflexión

DOI: http://dx.doi.org/10.18359/prole.1679

Forma de citación: Becerra, J. (2016). La relación de responsabilidad entre el Estado y los proveedores de servicios de telecomunicaciones. Revista Prolegómenos Derechos y Valores, 19, 37, 57-66. DOI: http://dx.doi.org/10.18359/prole.1679

\section{Resumen}

Este artículo pretende describir la relación que existe entre los proveedores de servicios de telecomunicaciones (PST) (operadores) y el Estado en los casos donde las actuaciones de la administración a través de medios digitales comportan una responsabilidad debido al papel que desempeñan los PST, como intermediarios y garantes del servicio de comunicaciones entre la administración y los ciudadanos. Hemos tratado de establecer en el desarrollo de la investigación que se puede presentar aparte de la responsabilidad civil de los PST, una responsabilidad imputable al Estado en cuanto a su función de vigilancia, una responsabilidad derivada de la falla en la prestación del servicio, y una en cuanto a que la administración delega en los PST la salvaguarda, custodia y manejo de la información de los ciudadanos.

\section{Palabras clave:}

Tecnologías de la información y las comunicaciones, derecho, responsabilidad del Estado, proveedores de servicios de telecomunicaciones (operadores), falla del servicio.

\section{THE RELATIONSHIP OF RESPONSIBILITY BETWEEN THE GOVERNMENT AND THE SUPPLIERS OF TELECOMMUNICATION SERVICES}

\begin{abstract}
Summary
This article tries to describe the relationship that exists between the suppliers of telecommunication services (STS) and the Government in situations in which the actions of the administration through digital media entail a responsibility due to the role that STS play, as intermediaries and guarantors

* Artículo resultado de la investigación "La responsabilidad del Estado por la utilización de las administraciones públicas de las nuevas tecnologías de la información y la comunicación (TIC)" (2013-2014), del Grupo de Investigación en Derecho Público y TIC (G-TICCY) de la Universidad Católica de Colombia. Bogotá, Colombia.

* Abogado. Especializado en Ciencia Política y en Derecho Internacional ambas de la Universidad de Barcelona, España y candidato a doctor en Derecho por la Universidad de Barcelona, España. Fue interno de la Oficina de Naciones Unidas para Asuntos del Espacio Exterior y exasesor de la Secretaría de la Comisión Colombiana del Espacio. Actualmente es miembro de la Federación Iberoamericana de Asociaciones de Derecho e Informática y del Grupo de Investigación en Derecho Público y TIC de la Universidad Católica de Colombia. Correo electrónico: jabecerrao@ucatolica.edu.co
\end{abstract}


of the communication service between administration and citizens. In the investigation development, we have tried to establish that it is possible to submit, apart from civil responsibilities of the STS, a responsibility imputable to the Government regarding its monitoring function, a responsibility derived from the failure in the provision of services, and another one related to the fact that the administration delegates on the STS the safety, custody and handling of the citizens information.

\section{Keywords:}

Information and communication technologies, rights, Government responsibility, providers of telecommunication services, service failure.

\section{A RELAÇÃO DE RESPONSABILIDADE ENTRE O ESTADO E OS PROVEDORES DE SERVIÇOS DE TELECOMUNICAÇÃO}

\section{Resumo}

Este artigo pretende descrever a relação que existe entre os provedores de serviços de telecomunicação (SDT) (operadores) e o Estado nos casos onde a atuação da Administração Pública através dos meios digitais possui responsabilidade ao se considerar o papel que desempenham os SDT como intermediários e garantidores de serviços de comunicação entre a administração pública e a sociedade. Procuramos estabelecer durante o desenvolvimento desta investigação que se pode apresentar, além da responsabilidade civil dos SDT, uma responsabilidade derivada da falha na prestação de serviços, e outra quando se considera que a administração pública delega aos SDT a garantia, custodia e manutenção das informações para a sociedade

\section{Palavras-chave:}

Tecnologia da informação e as telecomunicações, direito, responsabilidade do estado, provedores de serviços de telecomunicações (operadores), falhas nos serviço.

\section{Introducción}

La llegada de las nuevas tecnologías ha llevado a la humanidad a grandes cambios en la estructura social, replanteando las relaciones entre todos los individuos. Este replanteamiento debe sin lugar a dudas tener consecuencias sobre el ordenamiento jurídico del Estado, que no se han analizado de forma profunda o sencillamente no se tienen en cuenta todavía.

Aún es incierto el alcance que las nuevas tecnologías de la información y la comunicación (TIC) causarán en toda la organización jurídica de nuestro país, debido sobre todo al hecho de que se están implementando de manera acelerada a todos los niveles en la administración pública, sustentado en un plan agresivo que ordena mediante un documento Conpes, la creación e implementación de medios electrónicos para que el ciudadano y el Estado puedan interactuar.

Es el caso de la intermediación que hacen los proveedores de servicios de telecomunicaciones (PST) entre el ciudadano y la administración, y como se puede derivar de esta intermediación una responsabilidad estatal cuando la prestación del servicio falla y como resultado se da una interrupción de la comunicación entre estos individuos, que pueda acarrear la violación de derechos. Ejemplo de la imposibilidad de participación en una licitación pública por falla del sistema, cuando la culpa es imputable al operador. Una de las consecuencias que debe estudiarse es 
la que se ocasiona de las actuaciones del propio Estado, teniendo en cuenta que estas pueden causar un daño antijurídico a los demás sujetos con los que interactúa. Temas como la protección de los derechos fundamentales, la protección de los datos de carácter personal, la publicación de contenidos por parte de la administración y el papel de los PST cuando prestan los servicios en la actuación del Estado por medios electrónicos, o sirven de intermediarios para la prestación de los mismos, derivan en la necesidad del entendimiento desde una nueva óptica jurídica de la relación entre la responsabilidad y las actuaciones de la administración pública.

Este entendimiento de la responsabilidad estatal en sus actuaciones dentro de las TIC se debe concebir en un sentido amplio, donde las nuevas fuerzas interactúan a todos los niveles del ordenamiento jurídico, donde la vulneración de los derechos fundamentales genera responsabilidad y donde las actuaciones de las administraciones públicas se redefinen.

Dicha responsabilidad se ha formado principalmente gracias al conjunto de políticas públicas, leyes y decretos que se emitieron en los últimos quince años en Colombia y que generaron nuevas situaciones y relaciones jurídicas, que nos llevan a plantear la cuestión de la responsabilidad como un punto central referente al papel que tiene y tendrá el Estado a través de medios electrónicos.

Ya existe en la doctrina y la legislación, en particular en el ámbito internacional, el precedente de responsabilidad para los PST en materia penal, por la no prevención de la actuación que causa el daño antijurídico a los usuarios de sus servicios (Fernández, 2007) y que nos hace presumir que también hay responsabilidad de los PST en otro tipo de circunstancias cuando se asocian con la administración y los ciudadanos. Si esta existe, existirá por tanto la responsabilidad del Estado conexa a la de los PST, si estos proceden en su nombre o como intermediarios ${ }^{1}$.

1 En materia de responsabilidad penal de los operadores consúltese Morales (2001).
El caso que se estudia aquí está dado por la relación de los PST y el Estado y la responsabilidad generada. Esta enmarca las situaciones concretas en las cuales la administración actúa con las personas naturales y jurídicas por medios electrónicos, para lo que se necesita de intermediarios que faciliten o medien en el proceso, que intervengan en nombre o representación de la administración pública supliendo sus actuaciones, o cuando el Estado como garante del ordenamiento jurídico tiene el deber de regular y vigilar el cumplimiento de las funciones de los operadores y no lo hace.

Presentamos en este artículo algunos de los resultados obtenidos de la investigación sobre la responsabilidad del Estado por la utilización de las administraciones públicas de las TIC. En especial atención de la promoción y respeto de los derechos fundamentales en general; lo referente a la protección de los datos; las actuaciones administrativas respecto de los PST (operadores); y la generación y difusión de contenidos por la publicación de contenidos en Internet a través del gobierno en línea, realizada por el Grupo de Investigación en Derecho Público y TIC de la Universidad Católica de Colombia. Que tuvo como uno de sus objetivos analizar la actuación de los poderes públicos como operadores de telecomunicaciones estableciendo la relación de responsabilidad y garantías por el incumplimiento de obligaciones.

Para ello aplicamos el método deductivo que nos permitió conformar un esquema de investigación por capítulos, basándonos en el modelo de investigación documental para extractar los datos pertinentes sometiéndolos a revisión, reseña y descripción. Además, se interpretaron los documentos según los ejes temáticos adoptados $y$, se realizó una síntesis que dio cuenta de la construcción teórica de la investigación documental para llegar a las conclusiones.

Por otro lado, y con apoyo en la propia interactividad de los métodos de investigación, llevamos a cabo el método inductivo en casos particulares de estudio de caso, que soportaron y comple- 
mentaron el análisis documental, facilitándonos aproximarnos al marco jurídico dentro del que opera la responsabilidad y si existe un vínculo entre el Estado y los PST.

Veremos entonces cómo se puede precisar la relación entre el Estado y los PST mediante la definición de conceptos, los sujetos intervinientes, las fuentes, la responsabilidad de los operadores en la ley y la configuración de una posible responsabilidad del Estado.

\section{A. El sector de las tecnologías de la información y la comunicación y el derecho}

\section{El concepto de tecnologías de la infor mación y la comunicación en relación con los proveedores de servicios de telecomunicaciones}

La relación entre el Estado y los PST también está dada por la propia definición del concepto de TIC, y de esta manera poder establecer el campo de acción concreto en el que el Estado y los PST interactúan.

Cabe aclarar que ante diversas definiciones y aproximaciones de quienes prestan los servicios de telecomunicaciones como: operadores, prestadores de servicios de la sociedad de la información, proveedores de redes y servicios de telecomunicaciones, proveedores de servicios de comunicaciones y operadores de comunicaciones, hemos decidido que acogeremos un concepto mixto que se construyó a partir de estas definiciones y con sustento en la ley 1341/2009, en la resolución 202/2010 y 588/2010 del Ministerio de Tecnologías de la Información y las Comunicaciones y en la resolución 3066/2011 de la Comisión de Regulación de Comunicaciones, entendiéndolos aquí como proveedores de servicios de telecomunicaciones (PST).

Para ello nos basamos en el artículo 6 de la ley 1341/2009, por la cual el Gobierno Nacional delimitó los principios y conceptos sobre la sociedad de la información y la organización de las TIC; y que comprende a las TIC como: "el conjunto de recursos, herramientas, equipos, programas informáticos, aplicaciones, redes y medios, que permiten la compilación, procesamiento, almacenamiento, transmisión de información como: voz, datos, texto, video e imágenes".

Esta norma nos lleva a establecer que todas las actuaciones del Estado o de un particular que se encaminen al tratamiento de información propia o de los individuos por cualquier medio electrónico o físico, se pueden incluir como parte del sector TIC. Y ya no solo lo hecho a través de medios electrónicos, pero que para nuestro objeto de estudio tomaremos solo desde esta perspectiva.

En el artículo 9 la ley en cita nos expone quién puede ser considerado como parte del sector TIC: empresas que manufacturen, comercialicen o presten servicios que den como resultado productos que recojan, procesen, creen, transmitan o expongan datos o información por medios electrónicos. En el mismo artículo se fijan los parámetros que caracterizan a estos sujetos cuando se prestan servicios: "Para las industrias de servicios, los productos de esta industria deben estar diseñados para permitir la función de tratamiento de la información y la comunicación por medios electrónicos, sin afectar negativamente el medioambiente".

Aquí nos surge una inquietud. ¿Puede entonces el Estado ser parte del sector TIC? La administración pública no es una industria, pero es innegable que de la definición amplia de las TIC que la propia ley en el artículo 6 hace, no podemos más que incluir al Estado como parte del sector TIC y no solo de forma indirecta (compañías prestadoras de servicios que conformen su estructura), sino directamente como quiera que el Estado presta servicios o los delega en particulares, a los cuales este debe vigilar y controlar e, incluso, sobre los cuales encomienda funciones que le corresponden, haciendo a nuestro parecer que el concepto que la ley precisa esté limitado e incompleto. 


\section{B. El cuerpo normativo}

Existe un cuerpo político y legislativo que sustenta la búsqueda de la relación de responsabilidad entre los PST y el Estado y que nos sirve de soporte. Están los documentos que justifican el desarrollo de políticas públicas para la intervención del Gobierno en el sector y la transformación de sus actuaciones con los ciudadanos como el Conpes $^{2} 3072 / 2000$, conocido como Agenda de conectividad que estableció la justificación, los plazos y procedimientos para la inclusión de las TIC en Colombia y el papel que el Estado desempeñaría como constructor, promotor y sobre todo no solo como sujeto pasivo, sino como agente activo de este sector. Y el Conpes 3650/2010 que determina la importancia estratégica de la política pública del gobierno en línea, por la cual la administración nacional tiene la obligación de relacionarse con los ciudadanos por medios electrónicos, procurando la implementación de estas tecnologías en plazos precisos y de la que debe surgir una responsabilidad.

En el ámbito legislativo tenemos como primera medida la ley 527/1999 que reguló lo referente a la firma electrónica, complementada por el decreto 2364/2012; la ley 1480/2011 que establece el Estatuto del Consumidor y asigna funciones y medios en el sector TIC; la ley 1564/2012 que creó el nuevo Código General del Proceso $e$ incluye la regulación de las actuaciones judiciales del Estado por medios electrónicos; la ley 1437/2011 que concibe el nuevo Código de Procedimiento Administrativo y fija la utilización de medios electrónicos en el procedimiento administrativo; la ley 1266/2008 sobre habeas data; la ley 1581/2012 sobre protección de datos personales, la ley 1712/2014 sobre el acceso a la información pública y el decreto 2573/2014 sobre gobierno en línea.

En materia de operadores referimos la ley $1341 / 2009$, por la cual se resuelven principios

2 Estos son los documentos que expide el Consejo Nacional de Política Económica y Social adscrito al Departamento Nacional de Planeación y que fija los lineamientos de Colombia en temas de políticas públicas y su desarrollo. y conceptos sobre la sociedad de la información y la organización de las TIC, y además se crea la Agencia Nacional del Espectro, ente que se encarga de regular el espacio físico que permite las comunicaciones; también el decreto 2693/2012 que dispone los lineamientos generales de la estrategia del gobierno en línea y ayuda a reglamentar las leyes 1341/2009, 1450/2011 -esta última establece el plan de desarrollo del país para el periodo 2011-2014-y 1751/2015 sobre el plan de desarrollo 2014-2018; el decreto 1972/2003 del antiguo Ministerio de Comunicaciones, por el cual se fija el régimen unificado de contraprestaciones por concepto de concesiones, autorizaciones, permisos y registros en materia de telecomunicaciones y los trámites para su liquidación, cobro, recaudo y pago; y el decreto 4948/2012 del Ministerio de Tecnologías de la Información y las Comunicaciones, mediante el que se reglamenta la habilitación general para la provisión de redes y servicios de telecomunicaciones y el registro de TIC.

Pese a este gran esfuerzo legislativo, es notoria la falta de articulación entre las diferentes normas y el desconocimiento generalizado de las implicaciones y los alcances de estos preceptos y la responsabilidad que se puede derivar de las actuaciones que ordenan.

\section{Los sujetos que intervienen}

Así como existe un cuerpo normativo que sustenta el sector de las TIC, existen unos sujetos que para la ley son relevantes y que podemos distinguir como: los usuarios, los proveedores de aplicaciones, los proveedores de contenidos y los proveedores de redes y servicios de telecomunicaciones, definidos en la resolución 202/2010 del Ministerio de Tecnologías de la Información y las Comunicaciones, sustentado por el mandato que le confiere el artículo 6 de la ley 1341/2009, para que expida un conjunto de definiciones relativas al sector TIC, en concordancia con la regulación internacional ${ }^{3}$.

La regulación internacional en materia TIC que obliga a Colombia, es la que emana principalmente de la Unión 
Los usuarios son todas las personas naturales o jurídicas que consumen los servicios que utilizan TIC para brindar estos servicios. Los proveedores de aplicaciones son personas naturales o jurídicas que proporcionan servicios para aplicaciones por medios electrónicos. Los proveedores de contenidos son personas naturales o jurídicas que generan un contenido determinado dentro del sector TIC. Los proveedores de redes y servicios de telecomunicaciones (que los equiparamos a los PST) son personas jurídicas responsables de la operación de redes o provisión de servicios de telecomunicaciones.

Esto nos lleva a diferenciar entre tres actores principales en la relación de responsabilidad: ciudadanos, Estado y PST, los cuales actuarían de la siguiente manera: ciudadanos son los usuarios de los servicios y operadores los PST y el Estado, que podría tener una función mixta, bien como proveedor de aplicaciones, proveedor de contenidos o como un PST, según el caso.

Con respecto a la responsabilidad se precisa que los PST podrán a su vez actuar como proveedores de contenidos o de aplicaciones, pero deberá ser en mandato de la intermediación de las actuaciones del Estado en los medios electrónicos, evitando que dicha relación se considere entonces solo de carácter privado, pasando a ser una relación de derecho público, entre los usuarios y los operadores.

\section{De la responsabilidad}

La teoría de la responsabilidad estatal está ampliamente definida en nuestro ordenamiento, pues funda un marco jurídico que determina y le da validez a la responsabilidad de la administración pública. Por ejemplo, como mecanismo de protección de los administrados, a través de sentencias como la C-333/1996 de la Corte Constitucional colombiana y que reiteró la C-892/2001, estableciendo los elementos de la responsabilidad; en la sentencia del Consejo de

Internacional de Telecomunicaciones y de los tratados internacionales vigentes que ha suscrito Colombia con otros Estados.
Estado, expedientes 10948 y 11643 del 21 de octubre de 1999; en el tema de la cláusula general de responsabilidad estatal, con la sentencia de la Corte Constitucional colombiana, C-892/2001; y en cuanto a la finalidad de protección por parte de las autoridades públicas, en la sentencia del Consejo de Estado, AG-2001-213 del 26 de enero de $2006^{4}$.

Creemos que la perspectiva correcta de abordar el problema que planteamos es la responsabilidad subjetiva del Estado (Martínez, 1988), en los casos de la prestación de un servicio por parte de los PST. Entendiendo como lo ha dicho el Consejo de Estado, que esta responsabilidad no se excluye en nuestro ordenamiento actual, sino más bien se amplía, para abordar la responsabilidad en su conjunto y el daño antijurídico resultante (Nader, 2010).

El punto de partida es el artículo 90 de la Constitución Política de Colombia, el cual establece la orientación de la legislación actual sobre la responsabilidad basada en el daño antijurídico: "El Estado responderá patrimonialmente por los daños antijurídicos que le sean imputables, causados por la acción o la omisión de las autoridades públicas [...]". Así mismo debemos apoyarnos en el Consejo de Estado, que en su jurisprudencia conceptúa el daño antijurídico a partir de la doctrina española: "Un daño será antijurídico cuando la víctima del mismo no esté obligada por imperativo explícito del ordenamiento a soportar la lesión de un interés patrimonial garantizado por la norma jurídica" 5 (Consejo de Estado, 1991), para finalmente, determinar las condiciones que se deben tener en cuenta para su configuración: que sea un hecho injusto, efectivo, económicamente evaluable y susceptible de individualización personal o grupal (Consejo de Estado, 1991).

4 Para profundizar los términos, consúltese: Consejo de Estado, responsabilidad extracontractual del Estado, presupuestos, elementos, daño antijurídico, imputación, responsabilidad del Estado, constitucionalización y mecanismo de protección de los administrados.

5 Véase la sentencia del 22 de noviembre de 1991, Sala de lo Contencioso Administrativo, Sección Tercera, magistrado ponente Julio César Uribe Acosta. 
Esto nos lleva a plantear el problema jurídico: ¿existe responsabilidad del Estado por daños antijurídicos cuando los PST prestan los servicios de Internet a la administración pública?

Desde esta perspectiva creemos que sí existe responsabilidad del Estado, ya que es este el obligado a la prestación adecuada del servicio en su conjunto. Es decir, es una responsabilidad completa en cuanto a: primero, la omisión de la administración por los vacíos de ley o la no regulación, entendiendo que es el mismo Estado el que está forzado a ofrecer las garantías e instrumentos legales de las propias cargas que impone a los ciudadanos; y segundo, el deber de garantizar y responder cuando a los ciudadanos se les violen, o no se les brinde una adecuada protección de sus derechos por terceros que actúen en su nombre (caso de los PST) o cuando se les imponga a los ciudadanos el deber de recurrir a estos para su relación con la administración.

1. La responsabilidad de los proveedores de servicios de telecomunicaciones

La legislación colombiana decretó que el régimen jurídico de los PST serán las normas de derecho privado, dentro de los cuales se contendrán:

[...] todas las comunicaciones, los actos y los contratos, incluidos los relativos a su régimen laboral y las operaciones de crédito de los proveedores de las tecnologías de la información y las comunicaciones, cualquiera que sea su naturaleza, sin importar la composición de su capital [...] (ley 1341/2009, art. 55).

Entendiendo que sus actuaciones se deben a las personas jurídicas en el ámbito privado. Así pues, la ley les garantiza a los PST principios como la promoción y garantía de la libre competencia, pero les impone el deber de protección de los derechos de los usuarios, que avalaría que exista una responsabilidad de los operadores, cuando no se actúe de manera adecuada en la protección de tales derechos, pudiendo ser el caso de la falla o interrupción en la prestación del servicio, que produce consecuencias jurídicas.
De acuerdo con el principio de protección a los usuarios, la ley colombiana establece los principios de acceso, uso e interconexión (ley 1341/2009, art. 50), que le imprimen una obligación generadora de responsabilidad a los PST. Es compromiso de estos -respecto a los usuarios-, mantener y permitir el acceso, uso $e$ interconexión ${ }^{6}$ con cualquier otro operador, para garantizarle al usuario objetivos como los de trato no discriminatorio, transparencia o el no deterioro de la red en procesos de interconexión, que ayuden en últimas, a que los usuarios disfruten del servicio que se les brinda, sin importar la existencia de uno o más operadores o la relación de derecho privado que estos sustenten.

Es importante para la relación de responsabilidad, el artículo 50 de la ley 1341/2009, porque establece que la prestación del servicio de telecomunicaciones ofrecida por los PST, debe darse respetando la continuidad del mismo, sin poder invocar causales como las ya mencionadas. Es así como un operador es responsable de que el servicio se preste, y si no, de que el usuario pueda exigir una responsabilidad.

La legislación también implanta un régimen de sanciones por parte del Ministerio de Tecnologías de la Información y las Comunicaciones o por la entidad que esté facultada por la ley para ello (ley 1341/2009, art. 63), cuando se cometan infracciones a la confidencialidad de las comunicaciones, se provean redes o servicios que no estén previstos, se incumpla el acceso, uso, homologación e interconexión de las redes, no se cumpla con los parámetros de calidad y eficiencia, o se modifiquen los parámetros técnicos de las redes, afectándolas (ley 1341/2009, art. 64). Estos parámetros nos ayudan a establecer que los PST deberán responder por sus actuaciones cuando presten sus servicios al Estado, o cuando actúen en su nombre por delegación, convirtién-

\footnotetext{
6 Se define como interconexión: "A la vinculación de recursos físicos y soportes lógicos de las redes, incluidas las instalaciones esenciales, para permitir el funcionamiento de los servicios y/o aplicaciones y la interoperabilidad de las plataformas" (Ministerio de Tecnologías de la Información y las Comunicaciones, resolución 202/2009).
} 
dose en funcionarios públicos para estos efectos, como lo fija la ley 678/2001 en su artículo 2, parágrafo 1 , y por tanto sujetos incluso de una posible acción de repetición.

Se pueden presentar dos situaciones concretas que relacionan a los PST y al Estado, y las cuales suscitan una responsabilidad de la administración. La primera es la prestación misma del servicio de conexión y redes por medio de las cuales el Estado se vincula con los ciudadanos para llevar a cabo diversos trámites o procesos (notificaciones, presentación de documentos oficiales, expedición de certificados, licitaciones, etc.) y en los que el ciudadano o usuario podría exigir al Estado que provea o garantice una conexión permanente y fluida a través de la cual se le respeten sus derechos. Por lo que a pesar de ser una obligación del operador la prestación adecuada del servicio, debería ser también el Estado el que les garantice la comunicación idónea, pues es este, el que le impone la obligación a los individuos de relacionarse por este medio y no por ningún otro.

La segunda es cuando los PST proceden en representación o supliendo las funciones del Estado, o prestándole a este las herramientas necesarias para su relación con los ciudadanos. Se da cuando es un particular llamado operador el que presta el servicio de notificaciones, o de correos electrónicos de la administración, o realiza la gestión y administración de una página web del Gobierno, y por lo cual, no puede el Gobierno eximirse de su responsabilidad ante los ciudadanos, ni siquiera desde la figura de la contratación o cláusulas que exoneren de responsabilidad, ya que el PST es un mero intermediario y es la administración el sujeto que posee la responsabilidad.

\section{La responsabilidad del Estado}

La administración pública no ha tenido opción de elegir cómo relacionarse con los ciudadanos, esta viene dada principalmente por la ley y le impone como prioridad desarrollar y lograr la masificación de políticas públicas en la materia, como puede ser la estrategia del gobierno en línea ${ }^{7}$, que fija los fines y temas prioritarios para la masificación de esta iniciativa, y además, determina los porcentajes de penetración e implementación en municipios y departamentos ${ }^{8}$, así como las condiciones técnicas, administrativas, legales y financieras tendientes a su ejecución, por citar un ejemplo: "la provisión de trámites y servicios por múltiples canales y uso de tecnologías de información y comunicaciones en los procedimientos administrativos" (decreto 2693/2012). Es también la ley la que le imputa el principio de responsabilidad, por caso, la estrategia del gobierno en línea establecida en el decreto 2693/2012 en su artículo 3, da como principio y fundamento de este programa la responsabilidad consagrada en el artículo 209 de la Constitución Política, en el artículo 3 de la ley 489/1998 y en el artículo 3 de la ley 1437/2011, de los cuales el Gobierno no se puede abstraer.

La investigación nos llevó a establecer algunas de las situaciones donde el Estado podría ser responsable objetiva y subjetivamente (Arenas, 2013), cuando en sus actuaciones a través de medios electrónicos intervienen los PST. Este planteamiento se hace desde el reconocimiento de que la ley ha impuesto la obligación a los ciudadanos de relacionarse de forma electrónica con la administración y que para ello es menester que intervengan los PST. Esta relación está dada de facto, ya que son los operadores los que de uno u otro modo garantizan el acceso a Internet (medio usado para interactuar con la administración de manera electrónica) y en casos particulares, la propia administración les delega algunas de sus obligaciones para la prestación

\footnotetext{
"La estrategia gobierno en línea, liderada por el Ministerio de Tecnologías de la Información y las Comunicaciones, es el conjunto de instrumentos técnicos, normativos y de política pública que promueven la construcción de un Estado más eficiente, transparente y participativo, y que a su vez, preste mejores servicios con la colaboración de toda la sociedad mediante el aprovechamiento de la tecnología. Lo anterior con el fin de impulsar la competitividad y el mejoramiento de la calidad de vida para la prosperidad de todos los colombianos" (Estrategia Gobierno en Línea, s. f.).

8 Consúltese el documento Conpes 3650/2010.
} 
del servicio, obligándolos a fungir como administración, sin serlo.

Como explicamos en el título precedente, la responsabilidad por falla presunta del servicio se debería presentar por parte del Estado cuando, por ejemplo, conmina a sus ciudadanos a un proceso licitatorio donde intervienen medios electrónicos (decreto 2170/2012) y a responder, por ende, cuando no se le aseguren, por ejemplo, la participación en condiciones de igualdad a los licitantes (el caso de una plataforma electrónica que deja de funcionar por causa imputable al operador del servicio y por tanto le es imposible a una parte de los licitantes acceder al proceso). Podemos ver indicios claros ya en otras legislaciones como el caso español, que consagra el principio de igualdad y proporcionalidad en su ley de administración electrónica para evitar cualquier tipo de restricciones a los ciudadanos en su relación con la administración por medios electrónicos (Gamero, 2008).

Así mismo, no se puede eximir de responsabilidad al Estado cuando este delegue en los PST la custodia y salvaguarda de la información de los ciudadanos. (El expediente electrónico, en manos de operadores privados). Así se tramite a través de un contrato que exima de responsabilidad, pues en ese momento el operador o PST se erige en nombre de la administración y cumple las funciones que a este se le encomendaron por ley, configurándose la necesidad de vigilancia y control sobre los delegatarios elegidos, la culpa in vigilando. En estos casos la responsabilidad es de los PST, pero se extendería al Estado, como garante en el cumplimiento de las obligaciones que posee con los ciudadanos.

Por último, podemos evidenciar una responsabilidad del Estado por las fallas de control o regulación, sobre los servicios que prestan los proveedores de redes y servicios de telecomunicaciones que ocasionen un daño antijurídico, ya que cae sobre la administración la obligación de control como quiera que como lo expresa el Consejo de Estado9 ${ }^{9}$, si un particular debiera soportar individualmente una carga anormal y excepcional, constituiría un daño especial por el cual la administración debe responder (Rodríguez, 2013).

\section{Conclusiones}

Existe una relación de causalidad entre los servicios que prestan los PST y el actuar de la administración pública por medios electrónicos, que debe comportar una responsabilidad para el Estado sustentada en el mandato de la ley de que se creen y desarrollen actuaciones con los ciudadanos mediante estos canales, imponiéndoles la obligación de hacerlo.

El Estado debe garantizar tales actuaciones y no puede librarse de la responsabilidad establecida en la Constitución Política Nacional de 1991 y en su ordenamiento secundario, de tal suerte que las políticas públicas al respecto se deberán ejecutar observando las garantías de actuación del Estado, que protegen a los ciudadanos.

Los proveedores de redes y servicios de telecomunicaciones tienen una responsabilidad regida por las normas de derecho privado y generan una responsabilidad de la administración en el caso de la prestación misma del servicio de conexión y redes por medio de las cuales el Estado se debe relacionar con los ciudadanos, y cuando estos PST actúan en representación o supliendo las funciones del propio Estado, y una responsabilidad derivada de su propia actuación a su vez como prestadores del servicio de Internet.

Además de las responsabilidades que se derivan de la actuación de los proveedores, se presentan las directamente ligadas con la actuación del Estado, como la que surge de la presunta falla en el servicio, que se da consecuencia de la salvaguarda de información de particulares en manos de los proveedores de servicios y la que resulta de la falla en su control o regulación.

$9 \quad$ Véase la sentencia del Consejo de Estado del 28 de octubre de 1976, expediente 1.482 (708). 


\section{Referencias}

Alcaldía Mayor de Bogotá. (2008). Ley 1266. Alcaldía Mayor de Bogotá. (2011). Ley 1480. Alcaldía Mayor de Bogotá. (2014). Ley 1712.

Arenas, H. (2003). El régimen de la responsabilidad objetiva. Lineamientos generales de la responsabilidad objetiva en Colombia (1 ${ }^{\text {a }}$ ed.). Bogotá: Legis.

Comisión de Regulación de Comunicaciones \& Ministerio de Tecnologías de la Información y las Comunicaciones. (2011). Resolución 3066.

Congreso de la República de Colombia. (1991). Constitución Política.

Consejo de Estado. (1999). Sentencias 10948 y 11643.

Consejo de Estado. (2006). Sentencia AG2001-213.

Consejo Nacional de Política Económica y Social. (2000). Conpes 3072. Bogotá: Departamento Nacional de Planeación.

Consejo Nacional de Política Económica y Social. (2010). Conpes 3650. Bogotá: Departamento Nacional de Planeación.

Corte Constitucional Colombiana. (1996). Sentencia C-333.

Corte Constitucional Colombiana. (2001). Sentencia C-892.

Corte Constitucional Colombiana. (2009). Sentencia C-892.

Fernández, J. G. (2007). Las implicaciones penales de la LSSICE para los proveedores de servicios de Internet. En: L. Cotino (ed.). Libertades en Internet. La red y las libertades de expresión e información. Madrid: Tirant Lo Blanch. P. 387-411.

Gamero, E. (2008). Objeto, ámbito de aplicación y principios generales de la ley de administración electrónica: su posición en el sistema de fuente. En: E. Gamero \& J. Valero (eds.).
La ley de administración electrónica. Los principios de la administración electrónica. Madrid: Aranzadi. P. 57-115.

Martínez, G. (1988). La responsabilidad civil extracontractual en Colombia ( $4^{\mathrm{a}}$ ed.). Medellín: Biblioteca Jurídica Diké.

Ministerio de Comunicaciones. (2003). Decreto 1972.

Ministerio de Tecnologías de la Información y las Comunicaciones. (2009). Ley 1341.

Ministerio de Tecnologías de la Información y las Comunicaciones. (2010a). Resolución 202.

Ministerio de Tecnologías de la Información y las Comunicaciones. (2010b). Resolución 588.

Ministerio de Tecnologías de la Información y las Comunicaciones. (2011). Ley 1450.

Ministerio de Tecnologías de la Información y las Comunicaciones. (2012a). Decreto 2693.

Ministerio de Tecnologías de la Información y las Comunicaciones. (2012b). Decreto 4948.

Nader, R. (2010). Evolución jurídica de la responsabilidad extracontractual del Estado en Colombia. Revista Advocatus, Edición Especial, 15, pp. 55-71.

Presidencia de la República de Colombia. (2002). Decreto 2170, modificado por los decretos 2334/2006, 0066/2008, 2474/2008 y 734/2012. Proceso de contratación por medios electrónicos.

Presidencia de la República de Colombia. (2011). Ley 1437.

Presidencia de la República de Colombia. (2014). Decreto 2573.

Rodríguez, L. (2011). Derecho Administrativo. General y colombiano. 17 ed. Bogotá: Temis.

Senado de la República de Colombia. (2012). Ley 1564.

Superintendencia de Industria y Comercio. (1999). Ley 527.

Superintendencia de Industria y Comercio. (2012). Ley 1581. 